Agricultural Bioinformatics 

Kavi Kishor P.B. • Rajib Bandopadhyay

Prashanth Suravajhala

Editors

\section{Agricultural Bioinformatics}

照 Springer 


\section{Editors}

Kavi Kishor P.B.

Department of Genetics

Osmania University

Hyderabad, Andhra Pradesh, India

Prashanth Suravajhala

Bioclues Organization

IKP Knowledge Park, Picket

Secunderabad, Andhra Pradesh, India
Rajib Bandopadhyay

Department of Biotechnology

Birla Institute of Technology

Ranchi, Jharkhand, India

ISBN 978-81-322-1879-1

ISBN 978-81-322-1880-7 (eBook)

DOI 10.1007/978-81-322-1880-7

Springer New Delhi Heidelberg New York Dordrecht London

Library of Congress Control Number: 2014942334

\section{(C) Springer India 2014}

This work is subject to copyright. All rights are reserved by the Publisher, whether the whole or part of the material is concerned, specifically the rights of translation, reprinting, reuse of illustrations, recitation, broadcasting, reproduction on microfilms or in any other physical way, and transmission or information storage and retrieval, electronic adaptation, computer software, or by similar or dissimilar methodology now known or hereafter developed. Exempted from this legal reservation are brief excerpts in connection with reviews or scholarly analysis or material supplied specifically for the purpose of being entered and executed on a computer system, for exclusive use by the purchaser of the work. Duplication of this publication or parts thereof is permitted only under the provisions of the Copyright Law of the Publisher's location, in its current version, and permission for use must always be obtained from Springer. Permissions for use may be obtained through RightsLink at the Copyright Clearance Center. Violations are liable to prosecution under the respective Copyright Law.

The use of general descriptive names, registered names, trademarks, service marks, etc. in this publication does not imply, even in the absence of a specific statement, that such names are exempt from the relevant protective laws and regulations and therefore free for general use.

While the advice and information in this book are believed to be true and accurate at the date of publication, neither the authors nor the editors nor the publisher can accept any legal responsibility for any errors or omissions that may be made. The publisher makes no warranty, express or implied, with respect to the material contained herein.

Printed on acid-free paper

Springer is part of Springer Science+Business Media (www.springer.com) 


\section{Foreword}

Life on Earth would be unsustainable without plants. It has been repeatedly emphasized that plant biotechnology has the potential to provide our farmers with another green revolution. For graduate students who are studying agriculture and life sciences, it is imperative to recognize the knowledge and use of plant genomics. This information will help in the development of "new agriculture", and for the research community to drive technological advancement in agriculture. There is very high potential in plant research to translate genomics technology into agronomic advancement. However, this requires an understanding of computational biology, to comprehend biological data and phenomena through innovative applications of bioinformatics and biostatistics.

Bioinformatics focuses on developing and applying computationally intensive techniques such as data mining, pattern recognition, 3-dimensional visualization and machine learning, which helps to quickly and efficiently study large amounts of genomic information, chemical structure and other biological data, as well as to extract biological meaning from large data sets. During the past decade, there has been a tremendous increase in genomic tools available in key crop plants, including expressed sequence tags (ESTs), bacterial artificial chromosome (BAC) libraries and physical maps, genetic sequence polymorphisms, mutant collections and expression profiling resources. Whole genome sequences, well supported with genome annotations and information browsers to enable cross-genome comparisons, are now available for several reference plants, representing diverse families in dicots, monocots as well as lower plants. At the same time, genomic tools like next generation sequencing platforms are becoming increasingly more accessible and reasonably priced. This wealth and accessibility of new genomic resources has caused a paradigm shift wherein researchers whose motivation is crop improvement are no longer constrained by model systems.

Agricultural bioinformatics addresses agricultural problems directly. Genomic resources have created the means to speed the improvement of plants - agronomic, horticultural, and forest tree species. For example, this knowledge can contribute towards development of crops with desirable characteristics such as drought, disease and insect resistance, or crops that require less fertilizer and have higher nutritional content. Apart from plant and animal genomes, in silico biology encompasses the use of parasitic plant genomes and hundreds of available 'pathogen' genomes, including that of 
viruses, bacteria, phytoplasmas, protozoans, fungi and nematodes, providing an opportunity to study plant-pathogen interaction in order to enable disease diagnosis, management and cultivation of disease resistance transgenic crops.

It is truly an exciting time in agricultural research, and I think this book captures the scintillation of the evolving role of bioinformatics in agronomic advancement. With the advent of technological innovations through genomics and bioinformatics, many important questions of economic importance will now become increasingly addressable, bringing forth newer funding opportunities, and laying fruitful ground for many scientific careers.

I appreciate the efforts put in by Prof. Kavi Kishor P.B. and other contributors in bringing out this book.

National Institute of Plant Genome Research

Asis Datta

Jawaharlal Nehru University, New Delhi

National Institute of Plant Genome Research (NIPGR)

Aruna Asaf Ali Marg, JNU Campus

New Delhi-110067, India 


\section{Preface}

This book is a compact, cohesive review highlighting some important topics that streamline bioinformatics in a nutshell for the applications in agriculture. It is intended both to informed researchers and upper graduates who wish to take up Agricultural Bioinformatics. Wide and interesting topics have been invited from researchers practicing agriculture, primarily plant bioinformatics. The value of bioinformatics in general has caught interest for researchers worldwide for various reasons. One primary reason is the added value in predicting the outcome using in silico based approaches, further defusing the wet lab experiments. However, there is much more consensus to be reached using the tools of bioinformatics. The last decade has seen innumerable tools and databases developed from which applied bioinformatics research has been exploited in the agricultural sector. The contributors in this book we believe have brought some delightful reviews to read and showcased which can be majorly applied for agri computing research besides common applications to all practices of bioinformatics research.

Sameera Panchangam et al. in their review "EST derived proteins in plant genomes: Where are we heading?" focus upon the need of understanding the ESTs better especially for those genomes that are not completely sequenced. Comprehensive commentary has been dealt on these sequences that are used to build the plant expressed transcripts and on how de novo the functional repertoire can be established. Saikumar and Dinesh Kumar in their chapter brings out an ideal review on the plant microRNAs describing several bioinformatics platforms and approaches on rise that has led to discovery of plant miRNAs, validation and need for understanding miRNAs from their progenitor messenger RNAs (mRNAs) have arisen. With the introduction of sequence-specific miRNA signatures recently found, he discusses myriad of dimensions where miRNAs from oilseed crops are being associated with several putative functional and evolutionary events. Priyanka James et al. review Medbase, a compendium of medicinal plants. Bioinformatics approaches leveraging plant-based knowledge discovery has offered plethora of new tools for the identification of genes and pathways involved in the production of secondary metabolites. Their database is aimed at bioinformatics strategies associated with important ethnic medicinal plants and it helps to identify therapeutically important active compounds. They further suggest the use of this database in helping farmers involved in cultivating medicinal plants. 
Khyati and Sivaramaiah in their book chapter, titled "Plant-Microbial Interaction: A Dialogue between Two Dynamic Bioentities" discuss the role of ecology that plant-microbe association has influenced plant's diversity, metabolism, morphology, productivity, physiology, defense system and tolerance against adversities. Further, they discuss the omics approach being used to unveil the role of complex cryptic signaling process in the plant and microbes interaction. Sohini Gupta et al. discuss a brief overview of the viral silencing suppressors that have been identified in their chapter, titled "The Silent Assassins" and further delve on possibilities of the bioinformatics analyses of the viral silencing suppressors with specific case studies. They also conceptualize a future framework for application oriented use of these silent assassins. Subarna Thakur et al. in their chapter titled "Exploring the genomes of symbiotic diazotrophs with relevance to biological nitrogen fixation" discuss accessibility of new computational tools for genomic and proteomic analysis which have accelerated nitrogen fixation research predominantly in the areas of comparative genomics, protein chemistry and phylogenetic analysis of nitrogen fixation genes. Alternative phylogenetic approaches and protein structure based studies according to them have been quite prolific to divulge the unfamiliar aspects of symbiotic nitrogen fixation. Raj Pasam and Rajiv Singh in their chapter, titled "Association mapping A new paradigm for dissection of complex traits in crops" discuss the genetic basis of important agronomic traits and the scientific challenges and numerous efforts that are underway to understand and decipher the complexity of these traits in crops.

Katsumi Sakata et al. in their chapter, titled "Mining knowledge from omics data" give a gist of extracting knowledge from complex data in multiply layered biological information, a multiple omics-based approach would be a powerful method. They introduce a representative approach to integrate multiple omics data, and discuss topics that relate to mining knowledge from the omics data and further explain the meaning of the $p$-value and introduce applications of statistical testing to expression analyses for proteins specific to early seedling stage of soybean. Tiratha Raj Singh in his chapter, titled "Machine learning with special emphasis on support vector machines (SVMs) in systems biology: A plant perspective" discuss the progress of systems biology with integrative genomics and tools such as bioinformatics. He further narrates the role of recent developments in high-throughput techniques that led to the accumulation of a deluge of biological data. Logical applications from machine learning and how to deal with state-of-the-art techniques for managing data are discussed. Uma Devi et al. in their chapter on "Bioinformatic tools in the analysis of determinants of pathogenicity and ecology of entomopathogenic fungi used as microbial insecticides in crop protection" highlight the proteins involved in deciphering the effect of the inundative application of an entomopathogenic fungus on the native soil fungal diversity as extensively described in their works. The chapter highlights the bioinformatics bolstered investigation of the factors that influence the affectivity of insect pathogenic fungi as microbial biopesticides. Hima et al. describe in brief how bioinformatics can help in the task of understanding the genes associated with abiotic stress tolerance. Sujay 
Rakshit and Ganapathy in their review describe comparative genomics of cereal crops with special reference to Sorghum. Chavali et al. in their article address the need for cloud platforms with support for database design related virtualization enhancements. While discussing the designing databases specific for cloud in large enterprises, the authors describe database applications which need to be delivered using Cloud Platform while addressing SaaS (software-as-a-service) a dominant service model in cloud computing. The latter can be designed for end users and delivered over web.

We expect that all readers will find these articles interesting in this book; many might feel the lack of a livestock informatics article but we promise to bring that during our next edition.

Happy reading!

Hyderabad, India

Kavi Kishor P.B., Ph.D.

Ranchi, India Rajib Bandopadhyay, Ph.D.

Secunderabad, India Prashanth Suravajhala, Ph.D. 



\section{About the Editors}

Dr. Kavi Kishor P.B. is currently working as Emeritus Professor at the Department of Genetics, Osmania University, Hyderabad, India. He has 38 years of teaching, research and administrative experience. Published 160 papers including book chapters, produced 25 Ph.D.s and authored 5 books. He is a fellow of the National Academy of Sciences and also National Academy of Agricultural Sciences. Recipient of several gold medals, winner of Prof. Hiralal Chakravarthy Award from the ISCA, and the best teacher award from the Government of Andhra Pradesh. Received Rockefeller Foundation Fellowship from USA, Visiting Scientist Fellowship from Emory University, Atlanta, USA, Visiting Scientist Fellowship from Linkoping University, Sweden, and from Leibniz-Institute of Plant Genetics and Crop Plant Research, Gatersleben, Germany.

Dr. Rajib Bandopadhyay is working as Assistant Professor in the Department of Biotechnology, Birla Institute of Technology, Mesra, Ranchi, Jharkhand, India, for the last 8 years. He did his Ph.D. in Botany (Plant Molecular Biology) in 2004 from the University of Calcutta. He did his post-doctoral research work in Crop Biotechnology in India as well as in South Korea and USA. He has teaching experiences at the undergraduate and post graduate level in the field of Agriculture Biotechnology and also in Bioinformatics. Dr. Bandopadhyay is BOYSCAST fellowship recipient from DST, Government of India in 2007. He also participated in Southern Ocean Expedition in 2011. He has published more than 47 research papers in journals of international repute and authored a number of reviews, book chapters and manuals.

Dr. Prashanth Suravajhala is a Post-Doctoral Scientist and a virtual entrepreneur who founded Bioclues.org in 2005. He is also serving as an Associate Director of Bioinformatics.org, and has wide interests in lieu of functional genomics and systems biology of hypothetical proteins in human, specifically targeted to mitochondria. He loves mentoring undergraduates who want to pursue Bioinformatics. Dr. Suravajhala has completed his Ph.D. from Aalborg Universitet, Denmark. He has published 20 papers in international peer-reviewed journals. 



\section{Contents}

Association Mapping: A New Paradigm for Dissection

of Complex Traits in Crops . . . . . . . . . . . . . . . . . . . .

Raj K. Pasam and Rajiv Sharma

The Silent Assassins: Informatics of Plant

Viral Silencing Suppressors . . . . . . . . . . . . . . . . . . . . 2

Sohini Gupta, Sayak Ganguli, and Abhijit Datta

Tackling the Heat-Stress Tolerance in Crop Plants:

A Bioinformatics Approach . . . . . . . . . . . . . . . . 33

Sudhakar Reddy Palakolanu, Vincent Vadez,

Sreenivasulu Nese, and Kavi Kishor P.B.

Comparative Genomics of Cereal Crops:

Status and Future Prospects . . . . . . . . . . . . . . . 59

Sujay Rakshit and K.N. Ganapathy

A Comprehensive Overview on Application

of Bioinformatics and Computational Statistics

in Rice Genomics Toward an Amalgamated

Approach for Improving Acquaintance Base . . . . . . . . . . . . . . 89

Jahangir Imam, Mukesh Nitin, Neha Nancy Toppo,

Nimai Prasad Mandal, Yogesh Kumar, Mukund Variar,

Rajib Bandopadhyay, and Pratyoosh Shukla

Contribution of Bioinformatics to Gene Discovery

in Salt Stress Responses in Plants

P. Hima Kumari, S. Anil Kumar, Prashanth Suravajhala,

N. Jalaja, P. Rathna Giri, and Kavi Kishor P.B.

Peanut Bioinformatics: Tools and Applications

for Developing More Effective Immunotherapies

for Peanut Allergy and Improving Food Safety . . . . . . . . . . . . . 129

Venkatesh Kandula, Virginia A. Gottschalk,

Ramesh Katam, and Roja Rani Anupalli

Plant MicroRNAs: An Overview

Kompelli Saikumar and Viswanathaswamy Dinesh Kumar 
ESTs in Plants: Where Are We Heading?

Sameera Panchangam, Nalini Mallikarjuna,

and Prashanth Suravajhala

Bioinformatics Strategies Associated with Important

Ethnic Medicinal Plants

Priyanka James, S. Silpa, and Raghunath Keshavachandran

Mining Knowledge from Omics Data .

Katsumi Sakata, Takuji Nakamura, and Setsuko Komatsu

Cloud Computing in Agriculture

L.N. Chavali

Bioinformatic Tools in the Analysis

of Determinants of Pathogenicity and Ecology

of Entomopathogenic Fungi Used as Microbial

Insecticides in Crop Protection . . . . . . . . . . . . . . . . . . . 215

Uma Devi Koduru, Sandhya Galidevara, Annette Reineke,

and Akbar Ali Khan Pathan

Exploring the Genomes of Symbiotic Diazotrophs

with Relevance to Biological Nitrogen Fixation . . . . . . . . . . . . 235

Subarna Thakur, Asim K. Bothra, and Arnab Sen

Plant-Microbial Interaction: A Dialogue Between

Two Dynamic Bioentities .

Khyatiben V. Pathak and Sivaramaiah Nallapeta

Machine Learning with Special Emphasis

on Support Vector Machines (SVMs) in Systems Biology:

A Plant Perspective .

Tiratha Raj Singh

Xanthine Derivatives: A Molecular Modeling Perspective

Renuka Suravajhala, Rajdeep Poddar, Sivaramaiah Nallapeta,

and Saif Ullah 